\title{
NORMAL CRITERIA CONCERNING SHARING VALUES
}

\author{
QINGCAI ZHANG
}

\begin{abstract}
In this paper the normality criterions concerning sharing values are researched and the old Montel theorem and Bloch-Valiron theorem are improved.
\end{abstract}

\section{Introduction and main results}

According to Bloch's principle every condition, which reduces a meromorphic function in the plane to a constant, makes a family of meromorphic functions in a domain $G$ normal. Although the principle is false in general ([4]), many authors proved normality criteria for families of meromorphic functions by starting from Picard type theorems ([1], [7], [8]). It is also more interesting to prove normality criteria by using conditions knowing from sharing value theorems. W. Schwick ([5]) first proved a interesting result that a family of meromorphic functions in a domain is normal if in which every function shares three distinct finite complex numbers with its first derivative. And in the preface of his paper, he also pointed out from Nevanlinna's famous five point theorem that if each pair functions $f$ and $g$ of meromorphic functions of a family share five fixed values $a_{j}$, the set $f^{(-1)}\left(\left\{a_{j}\right\}\right)$ are independent from $f$ and the normality follows immediately from Montel's theorem. In fact the number of sharing values in the result in his paper preface need only three, which has been proved by D. Sun in [6], and also will be seen later on in this paper.

We continue to study this problem in this paper, and first introduce some notations as follows.

Let $D$ be a domain in the complex plane $\boldsymbol{C}, S$ be a nonempty set of $C \cup\{\infty\}$, $h$ be a meromorphic function in $D$ and $l$ be a positive integer. Put

$$
\bar{E}_{l)}(S, h)=\bigcup_{a \in S}\{z \mid h(z)-a=0, z \in D, \text { with zero multiplicity } \leq l\},
$$

where the multiple zero is only counted once time (ignoring multiplicity).

2000 Mathematics Subject Classification: 30D35, 30D45.

Keywords: Normality, meromorphic function, sharing value.

This work supported by the NNSF of China.

Received November 30, 2000; revised June 8, 2001. 
When $S$ contains only one element, i.e. $S=\{a\}$, we denote $\bar{E}_{l)}(a, h)=$ $\bar{E}_{l)}(\{a\}, h)$. While $l \rightarrow \infty$, we denote $\bar{E}(S, h)=\bar{E}_{+\infty)}(S, h)$ and $\bar{E}(a, h)=$ $\bar{E}_{+\infty)}(a, h)$. We have some following definitions:

Two meromorphic functions $f$ and $g$ in $D$ are said to share the set $S$ IM with multiplicity $\leq l$ if $\bar{E}_{l)}(S, f)=\bar{E}_{l)}(S, g)$; to share the set $S$ IM if $\bar{E}(S, f)=$ $\bar{E}(S, g)$; to share the value $a$ IM with multiplicity $\leq l$ if $\bar{E}_{l)}(a, f)=\bar{E}_{l)}(a, g)$; and to share the value $a$ IM if $\bar{E}(a, f)=\bar{E}(a, g)$.

It is assumed that the reader is familiar with the notations of Nevanlinna theory such that $T(r, f), m(r, f), N(r, f), \bar{N}(r, f), S(r, f)$ and so on ([2]). The following two theorems are well-known in normality theory of meromorphic functions.

Montel's TheOREM ([1], [2], [8]). Let $F$ be a family of meromorphic functions in a domain $D, a_{1}, a_{2}, a_{3}$ be three distinct complex numbers in $\boldsymbol{C} \cup\{\infty\}$, if for every function $f \in F, f \neq a_{j}(j=1,2,3)$ in $D$, then $F$ is normal.

Bloch-VALiron Theorem ([7], [8]). Let $F$ be a family of meromorphic functions in a domain $D, a_{j}(j=1,2, \ldots, q)$ be $q$ distinct complex numbers in $\boldsymbol{C} \cup\{\infty\}$. If for every function $f \in F$, the zeros of $f-a_{j}$ have multiplicity $\geq$ $l_{j}+1(j=1,2, \ldots, q)$ in $D$, where $l_{1}, l_{2}, \ldots, l_{q}$ are $q$ positive integers such that

then $F$ is normal.

$$
\sum_{j=1}^{q}\left(1-\frac{1}{l_{j}+1}\right)>2
$$

In this paper, we continue to research these problems, and obtain the following results that are improvement of the two famous theorems.

THEOREM 1. Let $F$ be a family of meromorphic functions in a domain $D, f_{0}$ be a meromorphic function in $D$ (possibly not included in $F$ ), $a_{j}(j=1,2, \ldots, q$ ) be $q$ distinct complex numbers in $\boldsymbol{C} \cup\{\infty\} . \quad b_{j}(j=1,2, \ldots, p)$ be also $p$ distinct complex numbers in $\boldsymbol{C} \cup\{\infty\}$. If for every function $f \in F$,

$$
\bigcup_{j=1}^{q} \bar{E}_{\left.l_{j}\right)}\left(a_{j}, f\right) \subseteq \bigcup_{j=1}^{p} \bar{E}\left(b_{j}, f_{0}\right)
$$

where $l_{1}, l_{2}, \ldots, l_{q}$ are $q$ positive integers satisfying (1), then $F$ is normal.

For stating briefly, throughout this paper we always use $F$ to denote a family of meromorphic functions in a domain $D, a_{1}, a_{2}, \ldots, a_{q}$ are $q$ distinct complex numbers in $C \cup\{\infty\}, l_{1}, l_{2}, \ldots, l_{q}$ are positive integers, which will not be defined again when they appear later on. In Theorem 1 , choose $f_{0} \in F, p=q, b_{j}=a_{j}$ $(j=1,2, \ldots, q)$, we have as a special case

THEOREM 2. If each pair $f$ and $g$ of $F$ share the value $a_{j}$ with multiplicity $\leq l_{j}$, i.e. $\bar{E}_{\left.l_{j}\right)}\left(a_{j}, f\right)=\bar{E}_{l_{j}}\left(a_{j}, g\right)(j=1,2, \ldots, q)$ in $D$, where $l_{1}, l_{2}, \ldots, l_{q}$ satisfying $(1)$, then $F$ is normal. 
When $\bar{E}_{\left.l_{j}\right)}\left(a_{j}, f\right)=\bar{E}_{\left.l_{j}\right)}\left(a_{j}, g\right)=\emptyset(j=1,2, \ldots, q)$ in Theorem 2, we get BlochValiron theorem.

While $l_{j} \rightarrow+\infty(j=1,2, \ldots, q)$ in $(1)$, then $q \geq 3$, we get from Theorem 2

THEOREM 3. If each pair $f$ and $g$ of $F$ share the three distinct value $a_{1}, a_{2}, a_{3}$ $\mathrm{IM}$, i.e. $\bar{E}\left(a_{j}, f\right)=\bar{E}\left(a_{j}, g\right)(j=1,2,3)$ in $D$, then $F$ is normal. in [6].

This theorem has been proved by D. Sun using Ahlfors geometrical method

When $\bar{E}\left(a_{j}, f\right)=\bar{E}\left(a_{j}, g\right)=\emptyset(j=1,2,3)$ in Theorem 3, we obtain Montel's Theorem.

If $l_{1}=l_{2}=\cdots=l_{q}=l$ in $(1)$, then $q>2+2 / l$. From Theorem 1 we also get

THEOREM 4. Let $l, q$ be two positive integers satisfying $q>2+2 / l$, if each pair $f$ and $g$ of $F$ share the set $S=\left\{a_{1}, a_{2}, \ldots, a_{q}\right\}$ IM with multiplicity $\leq l$, i.e. $\bar{E}_{l)}(S, f)=\bar{E}_{l)}(S, g)(j=1,2, \ldots, q)$ in $D$, then $F$ is normal.

As corollaries of Theorem 4, we have

COROLlary. If each pair $f$ and $g$ of $F$ share the set $S=\left\{a_{1}, a_{2}, a_{3}, a_{4}, a_{5}\right\}$ IM with single zeros in $D$, then $F$ is normal.

If each pair $f$ and $g$ of $F$ share the set $S=\left\{a_{1}, a_{2}, a_{3}, a_{4}\right\}$ IM with multiplicity $\leq 2$ in $D$, then $F$ is normal.

If each pair $f$ and $g$ of $F$ share the set $S=\left\{a_{1}, a_{2}, a_{3}\right\}$ IM with multiplicity $\leq 3$ in $D$, then $F$ is normal.

Especially while $l \rightarrow+\infty$ in Theorem 4 , we have

THEOREM 5. If each pair $f$ and $g$ of $F$ share the set $S=\left\{a_{1}, a_{2}, a_{3}\right\}$ IM, i.e. $\bar{E}(S, f)=\bar{E}(S, g)$ in $D$, then $F$ is normal.

Theorem 5 is further improvement of Montel's Theorem and Theorem 3.

Clearly the number of elements of sharing set in Theorem 5 is sharp from the family $\left\{e^{n z}\right\}$ is not normal but each pair of it share the set $\{0, \infty\}$ IM in the unit disk.

\section{Main lemmas}

Lemma 1 ([3]). Let $U(r)$ be a nonnegative, increasing function on an interval $\left[R_{1}, R_{2}\right]\left(0<R_{1}<R_{2}<+\infty\right), a, b$ be two positive constants satisfying $b>(a+2)^{2}$. If the inequality

$$
U(r)<a\left\{\log ^{+} U(\rho)+\log \frac{\rho}{\rho-r}\right\}+b
$$


holds for every pair of $r, \rho\left(R_{1}<r<\rho<R_{2}\right)$, then we have

$$
U(r)<2 a \log \frac{R_{2}}{R_{2}-r}+2 b .
$$

Lemma 2 ([2], [8]). A family $F$ of functions analytic or meromorphic in $D$ is normal if and only if the functions

$$
f^{\sharp}(z)=\frac{\left|f^{\prime}(z)\right|}{1+|f(z)|^{2}} \quad f \in F
$$

are uniformly bounded on each compact subset of $D$.

Lemma 3 ([9]). Let $F$ be a family of meromorphic functions in the unit disk $\Delta$, then $F$ is not normal at $z=0$ if and only if there exist a sequence $f_{n} \in F$, a point sequence $z_{n} \rightarrow 0$ and a positive sequence $\rho_{n} \rightarrow 0$ such that $g_{n}(\zeta)=f_{n}\left(z_{n}+\rho_{n} \zeta\right)$ converges locally and uniformly to a nonconstant meromorphic function $g(\zeta)$ in the plane.

\section{Proof of theorems}

We only need to prove Theorem 1 .

Without loss generality we can assume that all of $\left\{a_{1}, a_{2}, \ldots, a_{q}\right\}$ are finite values, otherwise we can choose a finite value $d \neq a_{1}, a_{2}, \ldots, a_{q}$ and turn to prove the normality of family $\{1 /(f-d)\}$ since the family $F$ is as same as $\{1 /(f-d)\}$ in the normality.

By contrary suppose that the family $F$ is not normal in $D$, then there exists at least one point $z_{0} \in D$ such that $F$ is not normal at $z_{0}$. Without loss of generality, we may suppose that $z_{0}=0$. By Lemma 3 , there exist a sequence $f_{n} \in F$, a point sequence $z_{n} \rightarrow 0$, and a positive sequence $\rho_{n} \rightarrow 0$ such that $g_{n}(\zeta)=f_{n}\left(z_{n}+\rho_{n} \zeta\right)$ tends to a nonconstant meromorphic function $g(\zeta)$ uniformly on each compact subset of the plane $\boldsymbol{C}$, i.e.

$$
g_{n}(\zeta)=f_{n}\left(z_{n}+\rho_{n} \zeta\right) \Rightarrow g(\zeta)
$$

therefore

$$
g_{n}^{\prime}(\zeta)=\rho_{n} f_{n}^{\prime}\left(z_{n}+\rho_{n} \zeta\right) \Rightarrow g^{\prime}(\zeta)
$$

Since $g(\zeta)$ is nonconstant, we may choose a point $\zeta_{0}$ such that

$$
g\left(\zeta_{0}\right) \neq 0, \infty, a_{1}, a_{2}, \ldots, a_{q} ; \quad g^{\prime}\left(\zeta_{0}\right) \neq 0, \infty .
$$

Set

$$
h_{n}(z)=f_{n}\left(z_{n}+\rho_{n} \zeta_{0}+z\right), \quad \phi_{n}(z)=f_{0}\left(z_{n}+\rho_{n} \zeta_{0}+z\right),
$$

then

$$
h_{n}(0)=f_{n}\left(z_{n}+\rho_{n} \zeta_{0}\right) \rightarrow g\left(\zeta_{0}\right), \quad h_{n}^{\prime}(0)=f_{n}^{\prime}\left(z_{n}+\rho_{n} \zeta_{0}\right) \rightarrow \infty,
$$


and from (5) so for sufficiently large $n$,

$$
h_{n}(0) \neq 0, \infty, a_{1}, a_{2}, \ldots, a_{q} ; \quad h_{n}^{\prime}(0) \neq 0, \infty .
$$

Let $\delta=\inf \{|z| \mid z \in \partial D\}$, since $z_{n}+\rho_{n} \zeta_{0} \rightarrow 0$, then for sufficently large $n$, $\left|z_{n}+\rho_{n} \zeta_{0}\right|<\delta / 4$, and $z_{n}+\rho_{n} \zeta_{0}+z \in D,\left|z_{n}+\rho_{n} \zeta_{0}+z\right|<(3 / 4) \delta$ when $|z|<\delta / 2$. Using the second fundamental theorem for $h_{n}(z)$ in $|z|<\delta / 2$, we have

$$
\begin{gathered}
(q-2) T\left(r, h_{n}\right)<\sum_{j=1}^{q} \bar{N}\left(r, \frac{1}{h_{n}-a_{j}}\right)+S\left(r, h_{n}\right), \\
S\left(r, h_{n}\right)=m\left(r, \frac{h_{n}^{\prime}}{h_{n}}\right)+\sum_{j=1}^{q} m\left(r, \frac{h_{n}^{\prime}}{h_{n}-a_{j}}\right)+q \log ^{+} \frac{2 q}{\sigma}+\log 2 \\
+\log \frac{1}{\left|h_{n}^{\prime}(0)\right|}+\sum_{j=1}^{q} \log \left|h_{n}(0)-a_{j}\right|,
\end{gathered}
$$

where $\sigma=\min _{1 \leq i<j \leq q}\left\{\left|a_{i}-a_{j}\right|\right\}$. Since

$$
\bar{N}\left(r, \frac{1}{h_{n}-a_{j}}\right) \leq \bar{N}_{l_{j}}\left(r, \frac{1}{h_{n}-a_{j}}\right)+\frac{1}{l_{j}+1} T\left(r, h_{n}\right)+\frac{1}{l_{j}+1} \log \frac{1}{\left|h_{n}(0)-a_{j}\right|},
$$

and considering $l_{1}, l_{2}, \ldots, l_{q}$ satisfying (1), we have further

$$
T\left(r, h_{n}\right)<C\left\{\sum_{j=1}^{q} \bar{N}_{\left.l_{j}\right)}\left(r, \frac{1}{h_{n}-a_{j}}\right)+\sum_{j=1}^{q} \log ^{+} \frac{1}{\left|h_{n}(0)-a_{j}\right|}+S\left(r, h_{n}\right)\right\} .
$$

Throughout this paper, $C$ is a constant independent of $h_{n}$, which are not possibly the same for each appearing. From (6) and (2), for sufficiently large $n$ we have in $|z|<\delta / 2$

$$
\sum_{j=1}^{q} \bar{N}_{l_{j}}\left(r, \frac{1}{h_{n}-a_{j}}\right) \leq \sum_{j=1}^{p} \bar{N}\left(r, \frac{1}{\phi_{n}-b_{j}}\right) \leq \sum_{j=1}^{p} \bar{N}\left(\frac{3}{4} \delta, \frac{1}{f_{0}-b_{j}}\right) .
$$

We denote $A\left((3 / 4) \delta, f_{0}\right)=\sum_{j=1}^{p} \bar{N}\left((3 / 4) \delta, 1 /\left(f_{0}-b_{j}\right)\right)$ which is a constant independent of $h_{n}$. So

$$
T\left(r, h_{n}\right)<C\left\{A\left(\frac{3}{4} \delta, f_{0}\right)+\sum_{j=1}^{q} \log ^{+} \frac{1}{\left|h_{n}(0)-a_{j}\right|}+S\left(r, h_{n}\right)\right\} .
$$

Using Nevanlinna logarithmic derivative lemma ([3, p. 36)] for $S\left(r, h_{n}\right)$, and noticing that for $x \in\left(0, e^{-1}\right)$

$$
\log x+\beta \log ^{+} \log ^{+} \frac{1}{x} \leq \log ^{+} x+\beta(\log \beta-1),
$$


here $\beta$ is a positive constant, we have for $0<r<\rho<\delta / 2$

$$
\begin{aligned}
T\left(r, h_{n}\right)<C & \left\{1+\sum_{j=1}^{q} \log ^{+}\left|h_{n}(0)-a_{j}\right|+\sum_{j=1}^{q} \log ^{+} \frac{1}{\left|h_{n}(0)-a_{j}\right|}+\log ^{+} \frac{1}{\left|h_{n}^{\prime}(0)\right|}\right. \\
& \left.+\log ^{+} \log ^{+} \frac{1}{\left|h_{n}(0)\right|}+\log ^{+} \rho+\log ^{+} \frac{1}{r}+\log ^{+} \frac{1}{\rho-r}+\log ^{+} T\left(\rho, h_{n}\right)\right\} .
\end{aligned}
$$

From (7) and (5), for sufficiently large $n$, we know for $0<r<\rho<\delta / 2$

$$
T\left(r, h_{n}\right)<C\left\{1+\log ^{+} \rho+\log ^{+} \frac{1}{r}+\log ^{+} \frac{1}{\rho-r}+\log ^{+} T\left(\rho, h_{n}\right)\right\} .
$$

Applying Lemma 1 for $\delta / 8<r<\rho<\delta / 2$, from (11) we can deduce that

holds for $\delta / 8<r<\delta / 2$, therefore

$$
T\left(r, h_{n}\right)<2 C_{1}\left\{1+\log \frac{\delta / 2}{(\delta / 2)-r}\right\}
$$

$$
T\left(\frac{\delta}{4}, h_{n}\right)<C
$$

For sufficiently large $n$, from $(8)$, we know $h_{n}(0) \neq \infty$. Let $z^{*}$ be a pole of $h_{n}$ in $|z|<\delta / 4$, then

$$
\log \frac{\delta / 4}{\left|z^{*}\right|} \leq N\left(\frac{\delta}{4}, h_{n}\right) \leq T\left(\frac{\delta}{4}, h_{n}\right) \leq C,
$$

so $\left|z^{*}\right| \geq \delta /\left(4 e^{C}\right)$. Making $C$ large enough such that $\delta /\left(4 e^{C}\right)<\delta / 4$, let $\delta_{1}=$ $\delta /\left(4 e^{C}\right)$, then $h_{n}$ is holomorphic in $|z|<\delta_{1}$, and

$$
\log ^{+} M\left(\frac{\delta_{1}}{2}, h_{n}\right) \leq \frac{\delta_{1}+\left(\delta_{1} / 2\right)}{\delta_{1}-\left(\delta_{1} / 2\right)} T\left(\delta_{1}, h_{n}\right)<C,
$$

so for sufficiently large $n, h_{n}$ are bounded uniformly in $|z|<\delta_{1} / 2$, therefore $\left\{h_{n}\right\}$ is normal in $|z|<\delta_{1} / 2$, and so $\left\{f_{n}\right\}$ is normal in $|z|<\delta_{1} / 4$. By Lemma 2, we know that $f_{n}^{\sharp}$ are bounded uniformly in $|z| \leq \delta_{1} / 8$. And from

$$
g^{\sharp}(\zeta)=\lim _{n \rightarrow \infty} \rho_{n} f_{n}^{\sharp}\left(z_{n}+\rho_{n} \zeta\right)=0,
$$

we deduce that $g(\zeta)$ is constant which is a contradiction with $g(\zeta)$ being a nonconstant meromorphic function. This completes the proof of Theorem 1 .

Acknowledgement. The author thanks the referee for his/her comments and thanks Professor Yongcheng Yin for his help.

\section{REFERENCES}

[1] D. Drasin, Normal families and the Nevanlinna theory, Acta Math., 122 (1969), 231-263.

[2] W. K. Hayman, Meromorphic Functions, Oxford Mathematical Monographs, Clarendon Press, Oxford, 1964. 
[ 3 ] H. Milloux, Les Fonctions Méromorphes et leurs Dérivées, Actualités Sci. Indust. 888, Hermann, Paris, 1940.

[ 4 ] L. A. Rubel, Four counterexamples to Bloch's principle, Proc. Amer. Math. Soc., 98 (1986), $257-260$

[5] W. Schwick, Sharing values and normality, Arch. Math. (Basel), 59 (1992), 50-54.

[6] D. Sun, On the normal criterion of share value, J. Wuhan Univ. Natur. Sci. Ed., 3 (1994), 912 (in Chinese).

[7] M. TsusI, Potential Theorey in Modern Function Theory, Maruzen, Tokyo, 1959.

[8] L. YANG, Value Distribution Theory, Springer-Verlag, Berlin, 1993.

[9] L. Zalcman, A heuristic principle in complex function theory, Amer. Math. Monthly, 82 (1975), 813-817.

INSTITUTE OF MATHEMATICS

ZHEJIANG UNIVERSITY

HANGZHOU, 310027

P. R. CHINA

(Current Address)

Department of Basic Courses

Shandong Finance Institute

JiNAN, 250014

P. R. CHINA 